\title{
MEANS AND HERMITE INTERPOLATION
}

\author{
ALAN HORWITZ
}

\begin{abstract}
Let $m_{2}<m_{1}$ be two given nonnegative integers with $n=m_{1}+m_{2}+1$. For suitably differentiable $f$, we let $P, Q \in \pi_{n}$ be the Hermite polynomial interpolants to $f$ which satisfy $P^{(j)}(a)=f^{(j)}(a), j=0,1, \ldots, m_{1}$ and $P^{(j)}(b)=f^{(j)}(b), j=0,1, \ldots, m_{2}, Q^{(j)}(a)=$ $f^{(j)}(a), j=0,1, \ldots, m_{2}$ and $Q^{(j)}(b)=f^{(j)}(b), j=0,1, \ldots, m_{1}$. Suppose that $f \in C^{n+2}(I)$ with $f^{(n+1)}(x) \neq 0$ for $x \in(a, b)$. If $m_{1}-m_{2}$ is even, then there is a unique $x_{0}, a<x_{0}<b$, such that $P\left(x_{0}\right)=Q\left(x_{0}\right)$. If $m_{1}-m_{2}$ is odd, then there is a unique $x_{0}, a<x_{0}<b$, such that $f\left(x_{0}\right)=\frac{1}{2}\left(P\left(x_{0}\right)+Q\left(x_{0}\right)\right)$. $x_{0}$ defines a strict, symmetric mean, which we denote by $M_{f, m_{1}, m_{2}}(a, b)$. We prove various properties of these means. In particular, we show that $f(x)=x^{m_{1}+m_{2}+2}$ yields the arithmetic mean, $f(x)=x^{-1}$ yields the harmonic mean, and $f(x)=x^{\left(m_{1}+m_{2}+1\right) / 2}$ yields the geometric mean.
\end{abstract}

Mathematics subject classification (2000): 26D10.

Key words and phrases: Mean, arithmetic mean, geometric mean, Hermite interpolation, Taylor polynomial mean.

\section{REFERENCES}

[1] Ravi P. Agarwal \& Patricia J. Y. Wong, Error inequalities in polynomial interpolation and their applications, Kluwer, 1993.

[2] Alan Horwitz, Means and Taylor Polynomials, Journal of Mathematical Analysis and Applications 149(1990), 220-235.

[3] Alan HoRwitz, Means and Averages of Taylor Polynomials, Journal of Mathematical Analysis and Applications 176(1993), 404-412.

[4] E. ISAACSON AND H. B. KelLeR, Analysis of Numerical Methods, Wiley, New York, 1966.

[5] E. B. LEACH AND M. C. SHOLANDER, Multi-variable Extended Mean Values, Journal of Mathematical Analysis and Applications 104(1984), 390-407.

[6] A. M. OstrowsKI, Solution of Equations in Euclidean and Banach Spaces , 3rd ed., Academic Press, New York and London, 1973.

[7] Josip PeCaric \& Patricia J. Y. Wong, Polynomial interpolation and generalizations of mean value theorem, Nonlinear Funct. Anal. Appl. 6 (2001), no. 3, 329-340.

[8] Marko Petkovšek, Herbert Wilf, Doron Zeilberger, A. K. Peters, $A=B$, Massachusetts, 1996.

[9] A. SPITZBART, A Generalization of Hermite's Interpolation Formula, American Mathematical Monthly, Vol. 67, No. 1. (Jan., 1960), pp. 42-46. 